\title{
The iliac crest in forensic age estimation: evaluation of three methods in pelvis $\mathrm{X}$-rays
}

\author{
Viola Bartolini ${ }^{1}$ - Vilma Pinchi ${ }^{1}$ - Barbara Gualco ${ }^{1} \cdot$ Stefano Vanin $^{2}$. \\ Giusto Chiaracane $^{3} \cdot$ Giovanni D'Elia $^{4} \cdot$ Gian-Aristide Norelli $^{1} \cdot$ Martina Focardi $^{1}$
}

Received: 14 September 2016 / Accepted: 14 June 2017 / Published online: 20 June 2017

(C) Springer-Verlag GmbH Germany 2017

\begin{abstract}
Age assessment in children and young adults is a relevant forensic issue. It is requested not only in evaluating criminal responsibility in youths without proper identification documents, often in relation to age thresholds, but also for release of a residency permit, and asylum seekers of minors. The analysis of the Risser sign and the iliac crest ossification process has been proposed as a useful tool for forensic age estimation. We evaluated the applicability of three different age estimation methods on a sample of 497 pelvic radiographs of Italian individuals between 10 and 25 years of age. Each method showed high reliability for both reproducibility and repeatability. The staging technique inspired by Kreitner and Kellinghaus methods (KK-MS) is easier than the Risser method in applicability, as it is not affected by the variations of ossification. We observed that all subjects who attained stage $3 \mathrm{c}$ of KK-MS and 5 of Risser Fr were $>14$ years, suggesting the benefits of these methods for that age threshold. The applicability of the area measurement method, inspired by Cameriere's approach, ranged between 12 and 20 years, but the statistical analysis showed only a moderate correlation
\end{abstract}

Viola Bartolini

vilma.pinchi@unifi.it

1 Department of Health Sciences, Section of Medical Forensic Sciences, University of Florence, Largo Brambilla 3, 50134 Florence, Italy

2 Department of Biological Sciences, School of Applied Sciences, University of Huddersfield, Queensgate, Huddersfield HD1 3DH, UK

3 Orthopedic Department, Azienda Ospedaliero-Universitaria Careggi, Largo Brambilla 3, 50134 Florence, Italy

4 Radiology Department, Azienda Ospedaliero-Universitaria Careggi, Largo Brambilla 3, 50134 Florence, Italy with age. In order to evaluate the possible use of this approach it is therefore necessary to clarify and exclude external factors influencing the parameter. In conclusion, the iliac crest ossification is of interest in age estimation for forensic purposes. The evaluation of the pelvis X-ray, in addition to the other common dental and skeletal methods, could become a useful supplementary tool in age estimation for the 14 year threshold in order to fulfill forensic-level requirements.

Keywords Forensic age estimation - Iliac crest ossification . Risser sign $\cdot$ Scheletric age

\section{Introduction}

Over the past decades, European countries have been coping with a growing influx of immigrants, many of whom lack appropriate identification documents [1]. Applications for asylum by unaccompanied minors have also risen [2], as well as the number of crimes committed by minors [3-5]. The demand for forensic age estimation in adolescents and young adults therefore has increased (in German-speaking countries, for example, the requests for forensic expert age evaluations rose 270\%, from 185 in 1996 to 500 in 2001 [6], and according to recent data from Berlin, 157 cases were requested in 2014, more than double the 73 cases requested in 2004 [7]).

Consequently, there have been a number of studies which focus on designing accurate and precise procedures [8] and methods for age estimation in order to find new approaches suitable for forensic purposes.

The study of the pelvis in cadavers has long been used for forensic purposes [9-20]. In the living, the analysis of the process of ossification and fusion of pelvic bones has mainly focused on the iliac crest. Following the theory of Risser [21], the pelvis has been studied extensively for clinical purposes, 
especially in assessing skeletal maturation in subjects affected by scoliosis.

Lately, the analysis of the Risser sign and the iliac crest ossification process has been suggested as a useful tool for forensic age estimation. However, it is not employed for forensic use due to the lack of a consistent number of studies.

Wittschieber et al. [22] were the first to study the applicability of the Risser method in both the USA (US) and French (Fr) [23] version for forensic purposes, in subjects between 10 and 30 years.

Despite the discrepancies of certain stages of ossification from Risser's theory, the authors conclude that both methods are acceptable for age estimation for forensic purposes. This is especially true for the age threshold of 14 years, and the Fr method extended to 16 years.

In 2013, Wittschieber et al. [24] published a study where they evaluated the functionality of iliac crest ossification in forensic age estimation, testing a new method, inspired by the classification in stages of Kreitner [25] and Kellinghaus [26] for the clavicle. The new staging system for the iliac crest demonstrated the possibility of a valid diagnosis in both age thresholds of 14 and 16 years.

Wittschieber et al. [27] presented an innovative approach for the evaluation of the ossification of the iliac crest, inspired by the Cameriere's method. Unlike the other staging methods, this new suggested approach is not based on a subdivision in stages but on measurements and ratios of certain areas. The ratio between the area of the iliac crest apophysis and the iliac wing was calculated and used for linear regression analyses. The study showed an $R^{2}$ (coefficient of determination) ranging between 0.20 and 0.38 (depending on sex and pelvic side), with a standard error of estimate, between 1.91 and 2.00 years.

The present study, therefore, aims to verify these results from a sample of Italian individuals. The authors test the reliability and applicability of three methods based on the study of conventional X-rays for forensic purposes. Given the limitations of using ionizing radiation on the pelvis for nonclinical purposes, this study might provide useful age estimation whenever a pelvic radiograph is available. Furthermore, the study may offer additional information in evaluating the existence of useful biological evidence for age estimating on the pelvis, that could be investigated using future methods based on radiation-free techniques.

\section{Materials and methods}

The original sample was made up of 497 pelvis X-rays, belonging to Italian individuals of both sexes aged between 10 and 25 years who were admitted to the Orthopedic Trauma Center of Careggi Hospital (Florence, Italy), from 1 January 2013 to 31 December 2014. The age range of the sample was set according to previous scientific studies, which reports that the iliac crest ossification process does not begin before age 12 and is completed by the maximum age of 24 [22, 24, 27-32]. Only digital X-rays in anteroposterior projection performed in supine patients were included in the study. Images belonging to subjects with a history of developmental bone disorders were excluded. Radiographs with inadequate projections, artifacts, overlapping of foreign materials or bowel loops, fractures involving the pelvis, or conditions such as scoliosis (evident from the X-ray but not self-reported in clinical history) were also discarded.

Out of 497 images, 143 were excluded (115 due to defects or artifacts that prevented adequate visualization of the pelvis, 28 because of bone disorders), resulting in a sample of 354 subjects, composed of 186 males and 168 females (Table 1).

Age estimation was assessed applying the following three methods:

1- Risser sign staging method, in both the USA and European (French) versions [22, 23] (Risser US and Risser Fr methods).

Risser classification is based on the theory that during ossification process, the development of the apophysis of the iliac crest along the ilium begins from the anteromedial margin, while the fusion of the apophysis of the iliac crest with the ilium starts from the posteromedial side.

In both Risser Us and Fr staging systems, the attribution of the ossification stage (in six stages from 0 to 5 ) is based on the evaluation of the progression of the ossification along the iliac crest.

In Risser US (R-US) classification, commonly used for clinical purposes in the USA, the iliac crest is divided into quarters, defining the next four stages $(1-4)$. Stage 0 corresponds to the absence of ossification; stages 1-4 are related to the progression of the ossification by the appearance to completion, while stage 5 corresponds to the fusion of the apophysis of the iliac crest with the ilium, from the beginning of the process (starting at the posteromedial side) to its completion.

The Risser Fr (R-Fr) version, used for clinical purposes especially in Europe, differs from the previous one as it provides for the division of the iliac crest in three parts (thirds), and the fusion of the apophysis of the iliac crest with the ilium is divided in two stages (stage $4=$ incomplete fusion of the apophysis of the iliac crest with the ilium; stage $5=$ complete fusion of the apophysis of the iliac crest with the ilium).

2- The Kreitner and Kellinghaus method was originally used to provide age estimation based on clavicle ossification, applying a staging system that included main stages (1-4) and a subdivision of stages 2 and 3 in substages $(a-c)$. The method was modified by Wittschieber et al. [24] that applied it to iliac crest maturation. This modification of the 
Table 1 Composition of the sample

\begin{tabular}{lll}
\hline Age (years) & \multicolumn{2}{l}{ Number of individuals } \\
\hline & Females & Males \\
10 & 7 & 3 \\
11 & 6 & 0 \\
12 & 10 & 8 \\
13 & 7 & 9 \\
14 & 6 & 15 \\
15 & 6 & 14 \\
16 & 13 & 17 \\
17 & 10 & 13 \\
18 & 17 & 13 \\
19 & 9 & 18 \\
20 & 11 & 14 \\
21 & 7 & 8 \\
22 & 17 & 15 \\
23 & 17 & 14 \\
24 & 10 & 12 \\
25 & 15 & 13 \\
Total & 168 & 186 \\
\hline & & \\
\hline
\end{tabular}

original Kreitner and Kellinghaus (KK) method for iliac crest, which provided "main stages and substages," is used here and is referred to in this paper as Kreitner and Kellingaus main stages and substages (KK-MS) system.

KK-MS classification describes eight stages and substages (1, 2a, 2b, 2c, 3a, 3b, 3c, 4) evaluated according to the progression of the ossification of the apophyseal nucleus and of the fusion of the iliac apophysis with the pelvic bone. In this classification, it is assumed that both of the two processes may begin at any point along the iliac crest without a precise progression.

3- A method that provides a measurement of areas on X-rays (method here named AM) [27] according to Cameriere's approach for age estimation in the living. This method requires the measurement of the area of the ossification center (s) of the iliac crest (ICA) and the area of the iliac wing (IW); ICA/IW ratio is used for linear regression analysis and the elaboration of a formula that allows an estimation of age.

In agreement with previous studies, the evaluation of the images, measurements, and manual segmentations was performed at a computer workstation provided with standard picture archiving and communication system (PACS).

The following information was available per each X-ray: date of birth, sex of the subject, and the date of the examination.

Each analysis method (R-Us, R-Fr, KK-MS, AM) was applied blindly and separately to both sides of the pelvis.
In order to apply the evaluation of area measurement method, the areas of the ossification centers of ICA and the area of the IWs were measured [27]. Then, ICA/IW ratio was calculated, and statistical analysis was performed.

\section{Statistical analysis}

The results of the Risser (US and Fr) and the KK-MS methods are reported as minimum, maximum, mean, standard deviation, and median, upper, and lower quartiles. For these methods, differences between males and females were analyzed using the Mann-Whitney $U$ test, while comparison between the left and right sides was assessed using the Wilcoxon test; significance was assumed at $p<0.05$.

For the ICA/IW, differences between the right and left sides were evaluated using a paired samples $t$ test, due to the normality of the data.

Statistical Package for the Social Sciences (SPSS) 22.0 (SPSS Inc., Chicago, IL, USA) was used in performing the statistical analysis.

Best fittings of the function ICA/IW vs age were generated using a linear function with OriginPro (OriginLab Corporation). Statistical Package for the Social Sciences (SPSS) 22.0 for Windows (SPSS Inc., Chicago, IL, USA) was used to perform statistical analysis. The significant threshold was set at 5 and $1 \%$.

To test intra-observer and inter-observer reproducibility, two random samples of 45 and $25 \mathrm{X}$-rays were re-examined, respectively, after 3 weeks and by a second observer. Reliability analyses were performed following Ferrante [33].

\section{Results}

\section{Risser method (US and Fr versions)}

Table 2 summarizes the results obtained by Risser's method on 354 subjects (168 females and 186 males), grouped per sex and side of the pelvis.

The differences observed in the mean values for the two sides are not statistically significant $(p>0.05)$ for results based on both R-US and R-Fr, so only the right side was considered for statistical analysis.

Stage 1, corresponding to the beginning of ossification, was observed, in both US and Fr method, at the minimum age of 12.85 years for females and 13.64 in males.

The minimum age of appearance of the last stage of US method was 12.52 years in females and 15.07 for males, bilaterally.

The same age was confirmed in the R-Fr for males, but it differed for females, where the last stage was observed at the minimum age of 15.29 in both sides. According to the R-US, females reached the final stage with an average 
Table 2 Synopsis of the results for the two versions of the Risser method (R-US and R-Fr) for the right side of the pelvis, expressed in years

\begin{tabular}{|c|c|c|c|c|c|c|c|c|c|}
\hline Stage & Sex & $N$ & Min & Max & Mean & $\pm \mathrm{SD}$ & Median & LQ & UQ \\
\hline \multicolumn{10}{|c|}{ Risser sign (US), right side } \\
\hline \multirow[t]{2}{*}{0} & Female & 17 & 10.43 & 13.35 & 11.50 & 0.92 & 11.32 & 10.70 & 12.12 \\
\hline & Male & 14 & 10.03 & 14.86 & 12.77 & 1.62 & 12.87 & 11.67 & 14.28 \\
\hline \multirow[t]{2}{*}{1} & Female & 1 & 12.85 & - & - & - & - & - & - \\
\hline & Male & 1 & 13.64 & - & - & - & - & - & - \\
\hline \multirow[t]{2}{*}{2} & Female & 5 & 12.43 & 14.82 & 13.47 & 0.87 & 13.40 & 12.76 & 14.11 \\
\hline & Male & 4 & 13.15 & 15.23 & 13.96 & 0.96 & 13.72 & 13.18 & 14.96 \\
\hline \multirow[t]{2}{*}{3} & Female & 3 & 12.54 & 13.04 & 12.75 & 0.26 & 12.66 & 12.54 & 12.66 \\
\hline & Male & 10 & 12.45 & 15.71 & 13.92 & 1.02 & 14.12 & 12.92 & 14.60 \\
\hline \multirow[t]{2}{*}{4} & Female & 10 & 12.24 & 16.78 & 14.79 & 1.64 & 15.08 & 13.34 & 16.14 \\
\hline & Male & 26 & 13.34 & 18.56 & 15.72 & 1.25 & 15.59 & 14.75 & 16.81 \\
\hline \multirow[t]{2}{*}{5} & Female & 132 & 12.52 & 25.95 & 20.79 & 3.27 & 20.95 & 18.24 & 23.51 \\
\hline & Male & 131 & 15.07 & 25.78 & 20.94 & 2.91 & 20.86 & 18.69 & 23.35 \\
\hline \multicolumn{10}{|c|}{ Risser sign (Fr), right side } \\
\hline \multirow[t]{2}{*}{0} & Female & 17 & 10.43 & 13.85 & 11.50 & 0.92 & 11.32 & 10.70 & 12.12 \\
\hline & Male & 14 & 10.03 & 14.86 & 12.77 & 1.62 & 12.87 & 11.67 & 14.28 \\
\hline \multirow[t]{2}{*}{1} & Female & 2 & 12.85 & 13.60 & 13.22 & 0.53 & 13.22 & 12.85 & \\
\hline & Male & 1 & 13.64 & - & - & - & - & - & - \\
\hline \multirow[t]{2}{*}{2} & Female & 6 & 12.43 & 14.82 & 13.24 & 0.84 & 13.07 & 12.60 & 12.75 \\
\hline & Male & 10 & 12.45 & 15.23 & 13.63 & 0.88 & 13.31 & 12.92 & 14.39 \\
\hline \multirow[t]{2}{*}{3} & Female & 11 & 12.74 & 16.78 & 14.59 & 1.70 & 14.77 & 12.54 & 16.00 \\
\hline & Male & 29 & 13.34 & 18.56 & 15.64 & 1.23 & 15.56 & 14.73 & 16.77 \\
\hline \multirow[t]{2}{*}{4} & Female & 21 & 12.52 & 20.45 & 16.57 & 2.08 & 16.39 & 15.07 & 18.08 \\
\hline & Male & 24 & 14.07 & 19.99 & 17.05 & 1.23 & 17.01 & 16.31 & 17.86 \\
\hline \multirow[t]{2}{*}{5} & Female & 111 & 15.29 & 25.94 & 21.59 & 2.81 & 22.20 & 19.18 & 23.86 \\
\hline & Male & 108 & 15.07 & 25.78 & 21.74 & 2.53 & 21.98 & 19.73 & 23.75 \\
\hline
\end{tabular}

Min minimum age, Max maximum age, $S D$ standard deviation, $L Q$ lower quartile, $U Q$ upper quartile age of about 0.25 years before the males; this difference decreased to 0.15 years for R-Fr. These differences between the sexes are not statistically significant $(p=0.679)$.

The $k$ Cohen's test revealed high intra-observer and interobserver agreement ( $k$ always $>0.88$ ).

Graphics in Fig. 1 shows the trend of the studied methods compared to the chronological age (Fig. 1).

\section{Main and substage classification derived from Kreitner and Kellinghaus methods}

Table 3 reports the results of the application of KK-MS for age estimation on 354 radiographs, as minimum, maximum, mean $\pm \mathrm{SD}$, median, lower, and upper quartile, for both males and females.

No differences in the minimum age of appearance of the stages were observed between the left and right side of the pelvis, except in $3 \mathrm{a}$ and $3 \mathrm{~b}$ stages in males.

Only one male was evaluated in 2a stage; therefore, the statistical analysis was not performed for this cohort.
The final stage (4) was observed at the minimum age of 15.29 years for females and 15.07 for males; the analysis of the mean values, as observed for the Risser method, did not reveal any significant differences between the sexes $(p=0.679)$.

The Cohen $k$ test revealed a high intra-observer and inter-observer agreement ( $k 0.91$ and 0.89 , respectively), highlighting the high repeatability and reproducibility of the method. The trend of the stage and the age is reported in Figs. 2 and 3.

\section{Area measurement method based on the Cameriere's method}

It was possible to measure the areas of iliac crest ossification according to AM method in only 98 of the 354 X-rays which made up the study (Table 4).

In the remaining $256 \mathrm{X}$-rays, the ICA could not be measured for either the ossification was complete (225 cases) or it had not yet started (31 cases) (Table 4); therefore, AM method was not applicable. 

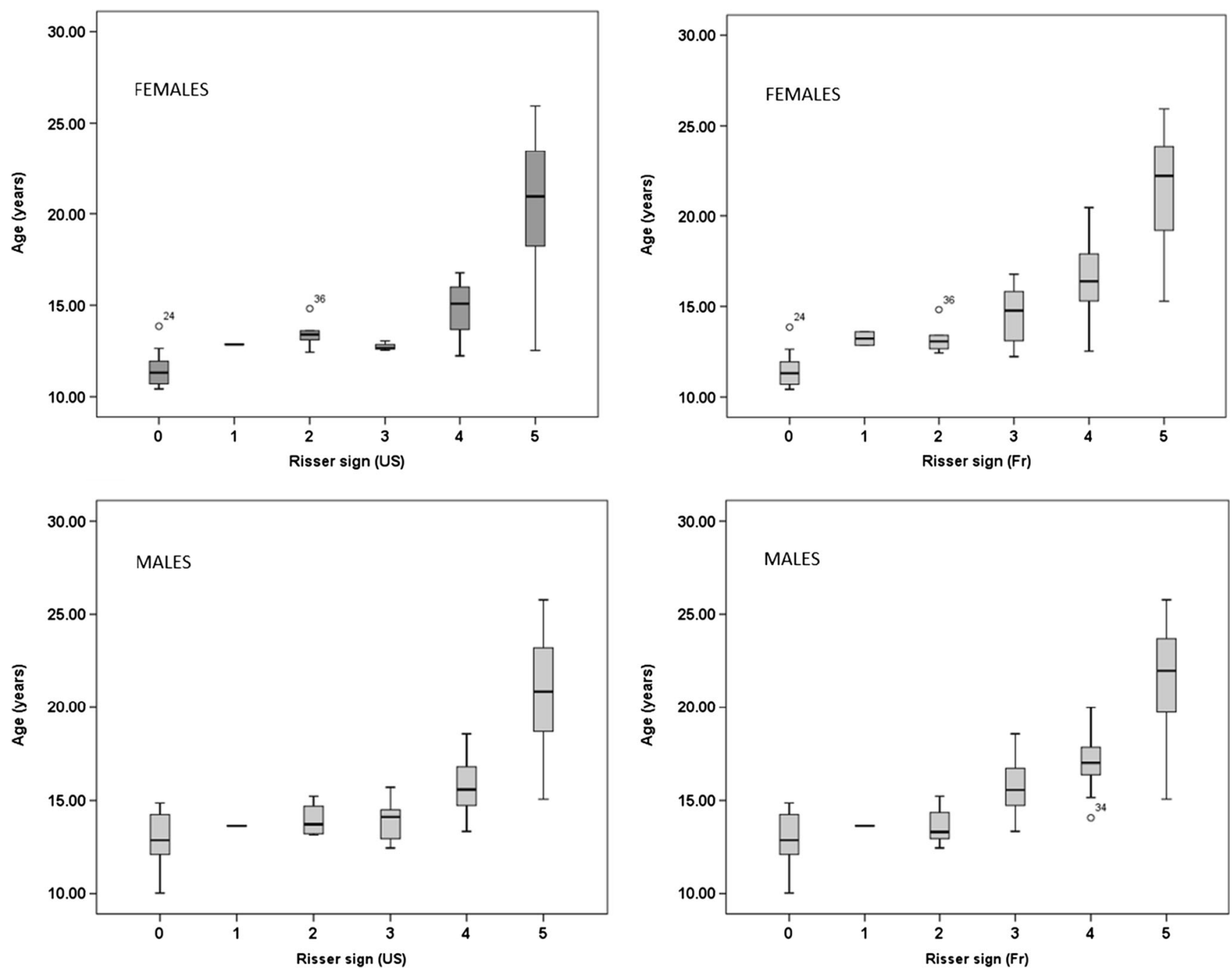

Fig. 1 Box plot for R-US and R-Fr in males and females

Incomplete ossification was observed in females between 12 and 20 years, while measurable areas were assessable up to 18 years in males (Table 4); complete ossification was observed at a minimum age of 15 years in both sexes (Table 4).

Figures 4 and 5 show the results for the left and right sides. Since no statistical significant differences were found between pelvic sides $(p=0.510)$, statistical analysis was performed on the right side, in accordance with Wittschieber et al. [27]

There were no statistically significant intra-observer and inter-observer differences between the paired sets of measurements done on the re-examined X-rays ( $\rho i>0.94)$ [respectively, $\rho i=0.94$ and 0.96 for both sides].

Statistical analyses indicated no differences between sexes $(p=0.35)$ and showed a weak positive correlation to age $(r=0.522)$. The calculated standard error was 1.93 years.
The relation between age and ICA/IW ratio was analyzed by a simple linear regression model, resulting in the following equation, applicable to both sexes:

Age $=13.345( \pm 0.381)$

$$
+17.838( \pm 2.978) \text { ICA/IW ratio }
$$

\section{Discussion}

Data coming out of the literature regarding the timing in ossification of the iliac crest is ambiguous. Consequently, forensic thresholds of interest for the application of pelvic age estimation methods are equally unclear. Striking differences emerge from the studies in data about both the starting age and the completion of fusion of the apophysis with the iliac crest. 
Table 3 Estimated age expressed in years applying KK-MS for the right pelvic side

\begin{tabular}{|c|c|c|c|c|c|c|c|c|c|}
\hline Stage & Sex & $N$ & Min & $\operatorname{Max}$ & Mean & $\pm \mathrm{SD}$ & Median & LQ & UQ \\
\hline \multicolumn{10}{|c|}{ KK-MS, right side } \\
\hline \multirow[t]{2}{*}{1} & Female & 17 & 10.43 & 13.85 & 11.50 & 0.92 & 11.32 & 10.70 & 12.12 \\
\hline & Male & 14 & 10.03 & 14.86 & 12.77 & 1.62 & 12.87 & 11.67 & 14.28 \\
\hline \multirow[t]{2}{*}{$2 \mathrm{a}$} & Female & 2 & 12.85 & 13.60 & 13.22 & 0.53 & 13.22 & 12.85 & - \\
\hline & Male & 1 & 13.64 & - & - & - & - & - & - \\
\hline \multirow[t]{2}{*}{$2 b$} & Female & 6 & 12.43 & 14.82 & 13.24 & 0.84 & 13.07 & 12.60 & 13.75 \\
\hline & Male & 12 & 12.45 & 15.71 & 13.85 & 1.01 & 13.74 & 13.00 & 14.46 \\
\hline \multirow[t]{2}{*}{$2 c$} & Female & 11 & 12.24 & 16.78 & 14.59 & 1.70 & 14.77 & 12.54 & 16.77 \\
\hline & Male & 26 & 13.34 & 18.56 & 15.75 & 1.025 & 15.59 & 14.75 & 16.81 \\
\hline \multirow[t]{2}{*}{$3 \mathrm{a}$} & Female & 2 & 12.52 & 16.39 & 14.45 & 2.74 & 14.45 & 12.52 & - \\
\hline & Male & 12 & 14.07 & 18.45 & 16.65 & 1.23 & 16.91 & 15.92 & 17.68 \\
\hline \multirow[t]{2}{*}{$3 b$} & Female & 13 & 13.13 & 20.37 & 16.43 & 1.93 & 16.36 & 15.06 & 17.87 \\
\hline & Male & 6 & 15.72 & 18.69 & 16.96 & 1.03 & 16.85 & 16.12 & 17.71 \\
\hline \multirow[t]{2}{*}{$3 c$} & Female & 6 & 14.84 & 20.45 & 17.58 & 1.98 & 17.93 & 15.64 & 18.90 \\
\hline & Male & 7 & 14.60 & 19.99 & 17.46 & 1.63 & 17.48 & 16.79 & 18.29 \\
\hline \multirow[t]{2}{*}{4} & Female & 111 & 15.29 & 25.94 & 21.58 & 2.82 & 22.20 & 19.18 & 23.86 \\
\hline & Male & 108 & 15.07 & 25.78 & 21.74 & 2.53 & 21.98 & 19.73 & 23.75 \\
\hline
\end{tabular}

Min minimum age, Max maximum age, $S D$ standard deviation, $L Q$ lower quartile, $U Q$ upper quartile
Among the studies, anthropological researches [29] generally indicate an older age when compared to the radiological ones [28, 30-32].

In the study by Wittschieber et al. [24], the mean age of onset and completion of the ossification of the iliac crest were, respectively, 14 and 24 in both sexes. The complete fusion (stages 4 of KK-MS and 5 according to R-Fr) was found at the minimum age of 17.90 in males and 16.42 in females. These results differ somewhat from our findings on the completion of bone maturation. This discrepancy affects the range of applicability of the methods.

Consistent with the previous study, using the AM method, apophysis of the iliac crest was observed at the minimum age of 12 years in both sexes. But we were able to define and

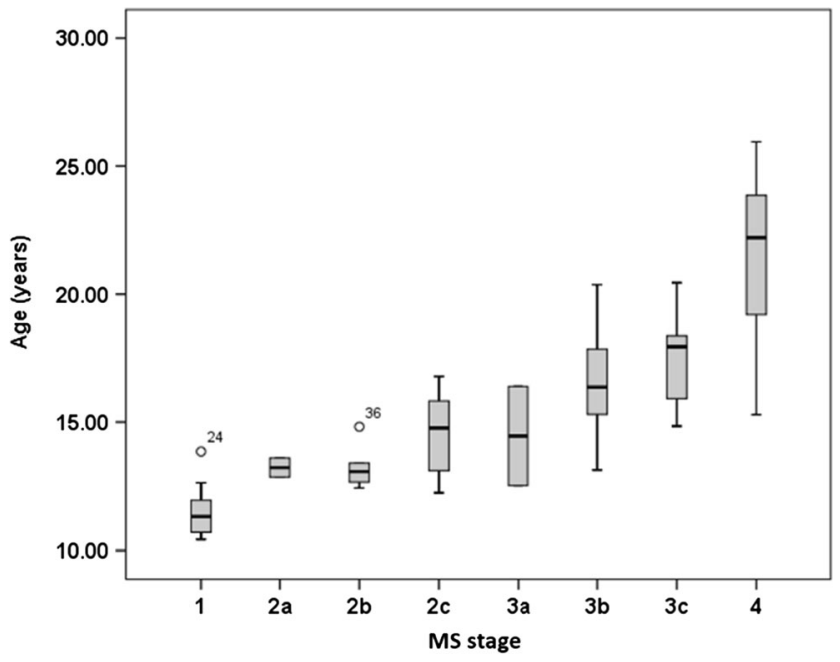

Fig. 2 Box plot of KK-MS in females calculate the area of the apophysis of the iliac crest and then apply the method only up to 18 years of age in males and 20 in females (instead of 24). But analyzing the threshold of applicability in close detail, it seems to be lower for females. All females over 17 years, with the exception of one aged 20, reached such an advanced level of ossification as to prevent the applicability of the method.

The minimum age of complete ossification (15.29 years for females and 15.07 for males) is lower compared to results reported by Wittschieber [24] and much lower compared to the anthropological studies.

This difference may be due to the difficulty of anteroposterior X-rays in viewing the posterior third of the

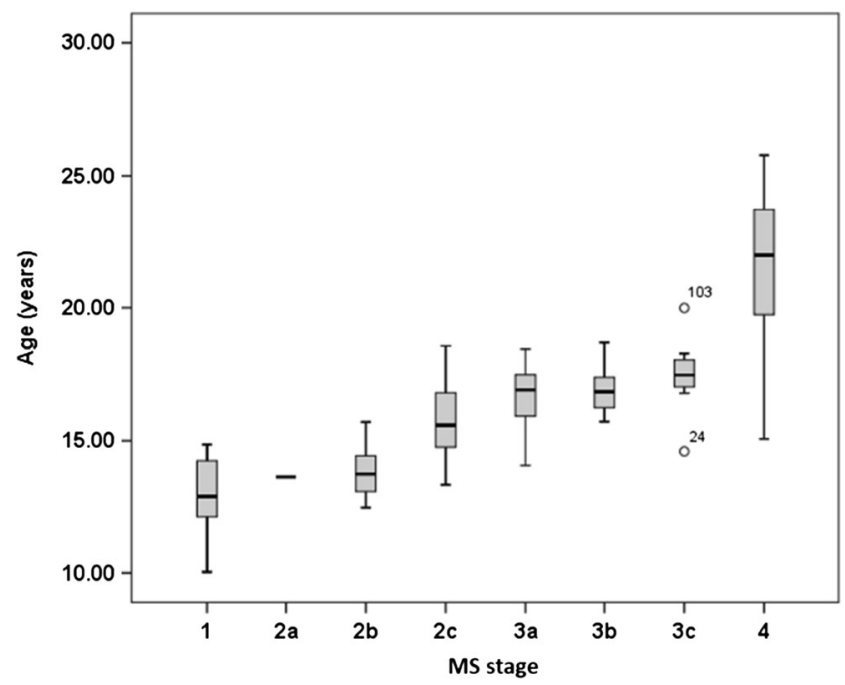

Fig. 3 Box plot of KK-MS in males 
Table 4 The number of subjects by gender and age cohort with incomplete iliac crest ossification (total amount of 98 subjects) for whom AM method was applicable (left column) and cases (256) impossible to be assessed (in the right side)

\begin{tabular}{lllll}
\hline & Incomplete ossification & X-rays non-assessable because of the stage of ossification \\
\hline Age (years) & No. of females & No. of males & No. of females & No. of males \\
10 & - & - & $7^{\mathrm{a}}$ & $3^{\mathrm{a}}$ \\
11 & - & - & $6^{\mathrm{a}}$ & - \\
12 & 7 & 3 & $3^{\mathrm{a}}$ & $5^{\mathrm{a}}$ \\
13 & 6 & 7 & $1^{\mathrm{a}}$ & $2^{\mathrm{a}}$ \\
14 & 6 & 11 & - & $4^{\mathrm{a}}$ \\
15 & 5 & 13 & $1^{\mathrm{b}}$ & $1^{\mathrm{b}}$ \\
16 & 7 & 15 & $6^{\mathrm{b}}$ & $2^{\mathrm{b}}$ \\
17 & 3 & 11 & $7^{\mathrm{b}}$ & $2^{\mathrm{b}}$ \\
18 & - & 3 & $17^{\mathrm{b}}$ & $10^{\mathrm{b}}$ \\
19 & - & - & $9^{\mathrm{b}}$ & $18^{\mathrm{b}}$ \\
20 & 1 & - & $10^{\mathrm{b}}$ & $14^{\mathrm{b}}$ \\
21 & - & - & $7^{\mathrm{b}}$ & $8^{\mathrm{b}}$ \\
22 & - & - & $17^{\mathrm{b}}$ & $15^{\mathrm{b}}$ \\
23 & - & - & $17^{\mathrm{b}}$ & $14^{\mathrm{b}}$ \\
24 & - & - & $10^{\mathrm{b}}$ & $12^{\mathrm{b}}$ \\
25 & - & 63 & $15^{\mathrm{b}}$ & $13^{\mathrm{b}}$ \\
Total & 35 & 133 & 123 \\
\hline
\end{tabular}

${ }^{a}$ The ossification has not already started

${ }^{\mathrm{b}}$ The fusion of the ICA with IW was already completed iliac crest apophysis. It has already been highlighted as a critical issue in the radiographic evaluation of Risser's sign for clinical purposes [34-36].

In accordance with Wittschieber findings [22] and other previous studies [37-39], and dissimilarly to the trend described by Risser, the appearance of the first center of epiphyseal ossification was not always at the anterolateral extremity of the iliac crest, but rather the posteromedial, and not infrequently in an intermediate position of the iliac crest curve. The

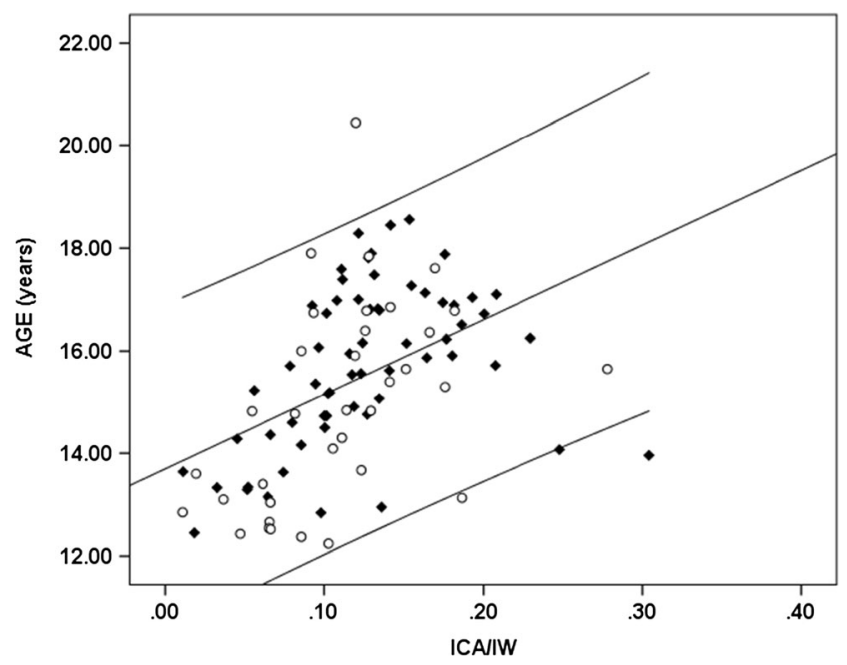

Fig. 4 Results for AM method applied to the left pelvic side. Circles represent females, diamonds males; the central line represents the best fitting; external ones correspond to the $95 \% \mathrm{CI}$ fusion of the ossification nucleus at times started in the central and lateral portion, and not at the posteromedial side.

This atypical pattern may be a source of error in the application of the Risser method. By contrast, it does not affect the application of KK-MS staging system, as the starting point of ossification or the origin of fusion is irrelevant in this approach.

A further critical issue which emerged in our study is the superposition of the abdominal organs with the iliac crest in $\mathrm{X}$-rays. This is a common limitation in all methods of radiological evaluation of the pelvis and was the reason in discarding a number of images. Other images were excluded due to the presence of diseases or artifacts that showed up on

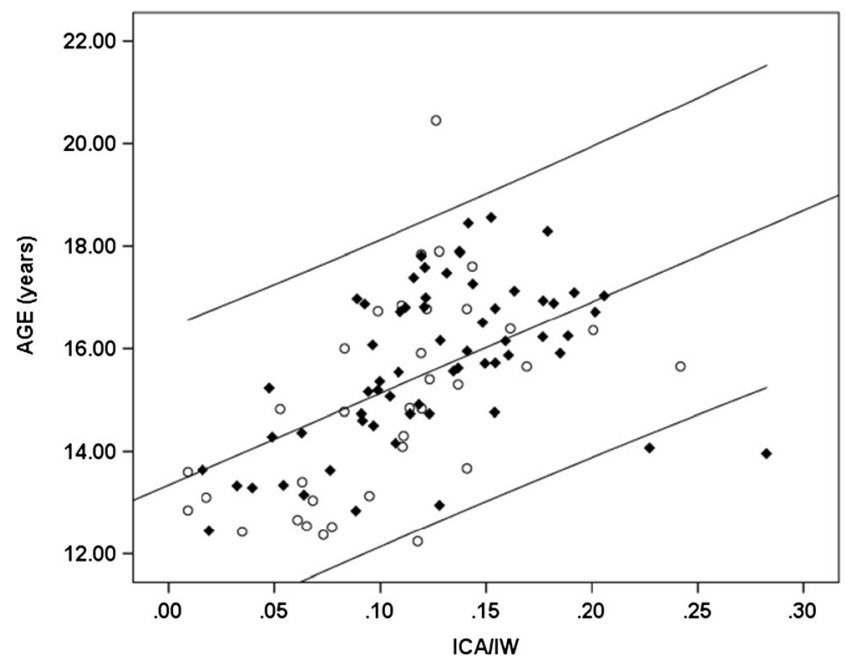

Fig. 5 Results for AM method applied to the right side 
the film. In part, this was because images were carried out under emergency conditions.

In spite of these limitations, the actual sample was adequate for the purposes of the study, even if AM method was studied using a smaller sample (98 rather than 354 X-rays). In a relevant number of cases, AM method turned out inapplicable because the ossification was not started or it was already completed, and both these conditions prevent the measurement of areas required in this approach.

Statistical analysis revealed good values of repeatability and reproducibility of all the methods.

The applicability of a forensic age estimation method, in a specific threshold, can be evaluated considering the minimum age of observation of each stage. Regarding this parameter, the application of the R-US was useless as the minimum age of observation for all stages, excepting males in stage V, was below the lowest relevant forensic threshold (14 years). More interesting findings concerned the application of the R-Fr, since the chronological age for all subjects of both sexes classified in stage 5 was superior to 14 years. According to Wittschieber, the 14 threshold could be even assessed for all subjects in stage $3 \mathrm{a}$.

The application of KK-MS proved to be interesting for 14 age threshold. All females classified in stage $3 \mathrm{c}$ were over 14.84 years, and males were at least 14.60 years old. Similarly, the same stage was observed by Wittschieber at the minimum age of 15 years.

Conversely, our study was not able to confirm the potential applicability of this method for the 16 year threshold, as previously pointed out by Wittschieber et al. Here, the authors described stage 4 only in males over 17 years and female over 16 . In our study, the same stage was observed around age 15 in both sexes, thus excluding an applicability for that threshold, so that Risser method was not interesting for 16 and 18 age thresholds.

The mean age was lower in females, at every stage. In advanced stages of ossification (4 and 5 of Us; $3-5$ of Fr; $3 b, 3 c$, and 4 of KK-MS) in both sexes, we observed a lower minimum age when compared to the Wittschieber et al. studies $[22,24]$. Although area measurement approach has been criticized because it is less immediate and more time consuming than other age estimation methods [40], it is actually easy to perform using common programs at any workstation PACs of radiology departments. In our study area, measurement method gained a Pearson correlation coefficient of 0.52 , in line with the Wittschieber's results [27] which found an $R^{2}$ between 0.20 and 0.38 . Our results are indicative of a moderate correlation to age; however, they are far worse than those reported by studies on dental mineralization [41], carpus ossification, and the epiphysis of the ulna and the radius [42-50].

As hypothesized by Wittschieber [27], this might be partially related to the anatomical iliac wing variability in X-rays. Moreover, as we tried standardizing our sample excluding all the images with evident artifacts or major dis-projections, it was however composed of radiographic images taken in the emergency room for clinical reasons, which explains why some minor dis-projections might have occurred in our sample.

If these critical issues had a minor relevance in the application of methods based on the attribution of stage (Risser and KK-MS), they would be far more influential in the AM method, which requires precision measurements of areas.

If the low correlation is confirmed, the error in age estimation would be not acceptable and the AM method inapplicable for forensic purposes. It is therefore essential to clarify whether the $R^{2}$ values depend on a bias in the sample or rather are due to an intrinsic limit of the method.

The effective dose of ionizing radiation from an X-ray of the pelvis $(700 \mu \mathrm{Sv})[51]$ is higher than the dose of an OPT $(26 \mu \mathrm{Sv})[52]$ and that of a X-ray of the clavicle $(220 \mu \mathrm{Sv})$ [53] or a CT scan of the medial clavicular epiphysis $(400 \mu \mathrm{Sv})$ $[51,54]$. Moreover, the exposure of abdominal and pelvic organs in young subjects raises ethical issues [55], which do not spare the other radiographic age estimation methods, and limits the possibility of performing such an exam for research, as well as for forensic purposes.

Given the ethical unacceptability of carrying out a prospective study which exposes to ionizing radiation, alternatively a retrospective analysis could be performed on a larger sample, selecting images with more precise and strict inclusion criteria.

According to the results of our study, further researches should be done in order to verify the effective fulfillment of forensic estimation requirements, focusing on the 14 age threshold. The radiographic study of the iliac crest ossification could in fact become a useful supplementary tool for age estimation in the living for the 14 threshold in addition to the other common dental and skeletal methods. But the ethical limits of radiographic exposition of the pelvis allow the radiographic study of the iliac crest ossification for age estimation only when a pelvic X-ray already exists.

However, the results of this study also support the opportunity for further research in the development of age assessment methods using non-radiographic investigations of the iliac crest. The primary studies in this direction, although limited, show intriguing results. The ultrasound examination was found to be a potential method applicable to the threshold for 14 and 16 years [56]. The use of MRIs resolves the problem of exposure to ionizing radiation and could remove some of the limitations of conventional radiography, also providing a better view of the posterior third of the iliac crest. The only study in the literature on the use of MRIs for age assessment on the iliac crest [57] specifically evaluates the applicability regarding the 20 year threshold in subjects aged 18 to 22 years. This suggests that further studies for lower age thresholds in a sample between 10 and 20 years are advisable.

The study of iliac crest ossification may also be applied for age estimation in the deceased. Cadavers and human remains anatomical structures can be viewed directly without interference and with the applicability of invasive procedures. 
However, the use of imaging methods for the study of the iliac crest ossification could find a useful application in autopsy investigations and identification procedures in cases of unidentified and/or damaged bodies when pelvises are intact. Finding incomplete ossification of the pelvis might allow an age range estimate of a subject and therefore provide valuable information for identification purposes.

\section{Conclusion}

Our study, which is preliminary due to the size and characteristics of the sample, highlights the following:

- The iliac crest ossification is of interest in age estimation for forensic purposes, both in the living and the deceased.

- Each of the methods evaluated in the study showed high reliability, for both reproducibility and repeatability.

- Considering the threshold of 14 years, that is relevant in most countries, we observed that all subjects (both females and males) who attained stage $3 \mathrm{c}$ of KK-MS and 5 of R-Fr were $>14$ years, suggesting that these $\mathrm{X}$-ray methods are useful for the 14 threshold, but less so for 16 years.

- The staging system with main and substages inspired by Kreitner and Kellinghaus method for the clavicle is easier to apply as it is not affected by the variations of ossification (area/s of iliac crest where ossification starts).

- The applicability of area measurement method ranged between 12 and 20 years. The statistical analysis showed very good reliability, but only showed a moderate correlation with age. In order to evaluate the usefulness of the method, it is therefore necessary to clarify and exclude external factors influencing the parameter, by enlarging the sample and applying more standardized procedures.

- Further studies should be performed in order to verify the fulfillment of forensic age estimation requirements and to develop radiation-free methods.

\section{References}

1. International Organization for Migration (IOM) World Migration Report 2011-Communicating effectively about migration https:// publications.iom.int/system/files/pdf/wmr2011_english.pdf Accessed 11 June 2017

2. European Migration Network, Policies on reception, return and integration arrangements for, and numbers of, unaccompanied minors. An EU comparative study, Bruxelles, 2010, www.emn. europa.eu. Accessed 11 June 2017

3. Ministero della Giustizia, Dipartimento Giustizia Minorile Ufficio I del Capo Dipartimento Servizio Statistica, I Servizi della Giustizia Minorile 15 ottobre 2016 Dati statistici https://www.giustizia.it/ resources/cms/documents/stat_dgm_servizi_giustizia_15ott2016_ mensile.pdf Accessed 11 June 2017
4. Gatti U, Fossa G, Bagnoli L, Binik O, Caccavale F, Cornelli R, D'Arrigo P, Di Nunno N, Greco O, Gualco B, Ravagnani L, Rensi R, Rocca G, Romano CA, Russo G, Traverso S (2015) Verde. Evolution and characteristics of the multi-centric research project "International Self-Report Delinquency Study": the contribution of Italian criminology. Ital J Criminol 3:164-168

5. Gualco B, Rensi R, Fossa G (2015) Family, parental presence and juvenile delinquency behaviour in Italy: data from a multicenter study performed by self-report questionnaires. Ital J Criminol 3: 177-194

6. Oehmichen M (2001) Geserick G Osteologische Identifikation und Altersschätzung. Lübeck, Schmidt-Römhild 26:255-261

7. Schmeling A, Dettmeyer R, Rudolf E, Vieth V, Geserick G (2016) Forensic age estimation: methods, certainty, and the law. Dtsch Arztebl Int 113(4):44-50. doi:10.3238/arztebl.2016.0044

8. Schmeling A, Grundmann C, Fuhrmann A, Kaatsch HJ, Knell B, Ramsthaler F, Reisinger W, Riepert T, Ritz-Timme S, Rösing FW, Rötzscher K, Geserick G (2008) Criteria for age estimation in living individuals. Int J Legal Med 122:457-460

9. Rissech C, Malgosa A (2005 Jan 29) Ilium growth study: applicability in sex and age diagnosis. Forensic Sci Int 147(2-3):165-174

10. Hanihara K, Suzuki T (1978) Estimation of age from the pubic symphysis by means of multiple regression analysis. Am J Phys Anthropol 48:233-239

11. Jackes MK (1985) Pubic symphysis age distributions. Am J Phys Anthropol 68:281-299

12. Brooks S, Suchey J (1990) Skeletal age determination based on the os pubis: a comparison of the Acsadi-Nemesk ' eri and Sucheybrooks methods. Hum Evol 5:227-238

13. Konigsberg LW, Herrmann NP, Wescott DJ, Kimmerle EH (2008) Estimation and evidence in forensic anthropology: age-at-death. J Forensic Sci 53:541-557

14. Hartnett KM (2010 Sep) Analysis of age-at-death estimation using data from a new, modern autopsy sample - part I: pubic bone. J Forensic Sci 55(5):1145-1151

15. Sinha A, Gupta V (1995) A study on estimation of age from pubic symphysis. Forensic Sci Int 75:73-78

16. Sharma G, Gargi J, Kalsey G, Singh D, Rai H, Sandhu R (2008) Determination of age from pubic symphysis: an autopsy study. Med Sci Law 48(2): 163-169

17. Lovejoy CO, Meindl RS, Pryzbeck TR, Mensforth RP (1985) Chronological metamorphosis of the auricular surface of the ilium: a new method for the determination of age at death. Am J Phys Anthropol 68:15-28

18. Buckberry JL, Chamberlain AT (2002 Nov) Age estimation from the auricular surface of the ilium: a revised method. Am J Phys Anthropol 119(3):231-239

19. Martins R, Oliveira PE, Schmitt A (2012) Estimation of age at death from the pubic symphysis and the auricular surface of the ilium using a smoothing procedure. Forensic Sci Int 219(1-3):287 e1-7

20. Schmitt A, Murail P, Cunha E, Rougé D (2002) Variability of the pattern of aging on the human skeleton: evidence from bone indicators and implications on age at death estimation. J Forensic Sci 47(6):1203-1209

21. Risser JC (1958) The iliac aphophysis: an invaluable sign in the management of scoliosis. Clin Orthop 11:111-119

22. Wittschieber D, Schmeling A, Schmidt S, Heindel W, Pfeiffer H, Vieth V (2013) The Risser sign for forensic age estimation in living individuals: a study on 643 pelvic radiographs. Forensic Sci Med Pathol 9:36-43

23. Bitan FD, Veliskakis KP, Campbell BC (2005) Differences in the Risser grading systems in the United States and France. Clin Orthop Relat Res 436:190-195

24. Wittschieber D, Vieth V, Domnick C, Pfeiffer H, Schmeling A (2013) The iliac crest in forensic age diagnostics: evaluation of 
the apophyseal ossification in conventional radiography. Int J Legal Med 127:473-479

25. Kreitner KF, Schweden FJ, Riepert T, Nafe B, Thelen M (1998) Bone age determination based on the study of the medial extremity of the clavicle. Eur Radiol 8(7):1116-1122

26. Kellinghaus M, Schulz R, Vieth V, Schmidt S, Pfeiffer H, Schmeling A (2010) Enhanced possibilities to make statements on the ossification status of the medial clavicular epiphysis using an amplified staging scheme in evaluating thin-slice CT scans. Int J Legal Med 124(4):321-325

27. Wittschieber D, Vieth V, Wierer T, Pfeiffer H, Schmeling A (2013) Cameriere's approach modified for pelvic radiographs: a novel method to assess apophyseal iliac crest ossification for the purpose of forensic age diagnostics. Int J Legal Med 127(4):825-829

28. Black S, Aggrawal A, Payne-James J. (2010) Age estimation in the living: the Practitioner's guide. Wiley-Blackwell, pp 284-289.

29. Webb PA, Suchey JM (1985) Epiphyseal union of the anterior iliac crest and medial clavicle in a modern multiracial sample of American males and females. Am J Phys Anthropol 68(4):457-466

30. Pratje A (1934) Über die Ossifikation des Beckens. Verh Anat Ges (Jena) 42:53-61

31. Diedrichs V, Wagner UA, Seiler W, Schmitt O (1998) Reference values for development of the iliac crest apophysis (Risser sign). Z Orthop Ihre Grenzgeb 136(3):226-229

32. Coqueugniot H, Weaver TD (2007) Brief communication: infracranial maturation in the skeletal collection from Coimbra, Portugal: new aging standards for epiphyseal union. Am J Phys Anthropol 134(3):424-437

33. Ferrante L, Cameriere R (2009) Statistical methods to assess the reliability of measurements in the procedures for forensic age estimation. Int J Legal Med 123:277-283

34. Izumi Y (1995) The accuracy of Risser staging. Spine (Phila Pa 1976) 20(17):1868-1871

35. Shuren N, Kasser JR, Emans JB, Rand F (1992) Reevaluation of the use of the Risser sign in idiopathic scoliosis. Spine (Phila Pa 1976) 17(3):359-361

36. Kotwicki $\mathrm{T}$ (2008) Risser sign: the value of the lateral spinal radiograph to assess the excursion of the iliac apophysis. Stud Health Technol Inform 140:44-47

37. Kotwicki T (2008) Improved accuracy in Risser sign grading with lateral spinal radiography. Eur Spine J 17:1676-1685

38. Zaoussis AL, James JIP (1958) The iliac apophysis and the evolution of curves in scoliosis. J Bone J Surg 40-B(3):442-453

39. Yang J, Bhandarkar A, Suh S, Hong J, Hwang J, Ham C (2014) Evaluation of accuracy of plain radiography in determining the Risser stage and identification of common sources of errors. $\mathrm{J}$ Orthop Surg Res 9(1):101

40. Thevissen PW, Fieuws S, Willems G (2011) Third molar development: measurements versus scores as age predictor. Arch Oral Biol 56:1035-1040

41. Cameriere R, Ferrante L, Belcastro MG, Bonfiglioli B, Rastelli E, Cingolani M (2007) Age estimation by pulp/tooth ratio in canines by mesial and vestibular peri-apical X-rays. J Forensic Sci 52(5): $1151-1155$

42. Cameriere R, Ferrante L, Ermenc B, Mirtella D, Strus K (2008) Age estimation using carpals: study of a Slovenian sample to test Cameriere's method. Forensic Sci Int 174(2-3):178-181

43. Cameriere R, Ferrante L (2008) Age estimation in children by measurement of carpals and epiphyses of radius and ulna and open apices in teeth: a pilot study. Forensic Sci Int 174(1):60-63

44. Cameriere R, Ferrante L, Mirtella D, Cingolani M (2006) Carpals and epiphyses of radius and ulna as age indicators. Int J Legal Med 120(3):143-146

45. Pinchi V, De Luca F, Focardi M, Pradella F, Vitale G, Ricciardi F, Norelli G-A (2016) Combining dental and skeletal evidence in age classification: pilot study in a sample of Italian sub-adults. Legal Med 20:75-79

46. Pinchi V, De Luca F, Ricciardi F, Focardi M, Piredda V, Mazzeo E, Norelli GA (2014) Skeletal age estimation for forensic purposes: a comparison of GP, TW2 and TW3 methods on an Italian sample. Forensic Sci Int 238:83-90

47. Corradi F, Pinchi V, Barsanti I, Garatti S (2013) Probabilistic classification of age by third molar development: the use of soft-evidence. J Forensic Sci 58(1):51-59

48. Pinchi V, Norelli G-A, Caputi F, Fassina G, Pradella F, Vincenti C (2012) Dental identification by comparison of antemortem and postmortem dental radiographs: influence of operator qualifications and cognitive bias. Forensic Sci Int 222(1-3):252-255

49. Pinchi V, Norelli G-A, Pradella F, Vitale G, Rugo D, Nieri M (2012) Comparison of the applicability of four odontological methods for age estimation of the 14 years legal threshold in a sample of Italian adolescents. J Forensic Odontostomatol 30(2):17-25

50. Pinchi V, Forestieri AL, Calvitti M (2007) Thickness of the dental (radicular) cementum: a parameter for estimating age. J Forensic Odontostomatol 25(1):1-6

51. Wall BF, Hart D (1997) Revised radiation doses for typical x-ray examinations. Brit J Radiol 70:437-439

52. Frederiksen NL, Benson BW, Sokolowski TW (1994) Effective dose and risk assessment from film tomography used for dental implant diagnostics. Dentomaxillofac Radiol 23(3):123-127

53. Okkalides D, Fotakis M (1994) Patient effective dose resulting from radiographic examinations. Br J Radiol 67(798):564-572

54. Schmeling A, Dettmeyer R, Rudolf E, Vieth V, Geseric G (2016) Forensic age estimation; methods, certainty, and the law. Dtsch Arztebl Int 113(4):44-50

55. Focardi M, Pinchi V, De Luca F, Norelli GA (2014) Age estimation for forensic purposes in Italy: ethical issues. Int J Legal Med 128(3): 515-522

56. Schmidt S, Schmeling A, Zwiesigk P, Pfeiffer H, Schulz R (2011 Mar) Sonographic evaluation of apophyseal ossification of the iliac crest in forensic age diagnostics in living individuals. Int $\mathrm{J}$ Legal Med 125(2):271-276

57. Wittschieber D, Vieth V, Timme M, Dvorak J, Schmeling A (2014 Jun) Magnetic resonance imaging of the iliac crest: age estimation in under-20 soccer players. Forensic Sci Med Pathol 10(2):198-202 